\title{
Effect of 2-hydroxy-4-(methylthio) butanoate (HMTBa) supplementation on rumen bacterial populations in dairy cows when exposed to diets with risk for milk fat depression
}

\author{
D. W. Pitta, ${ }^{1 *} \odot$ N. Indugu, ${ }^{1}$ B. Vecchiarelli, ${ }^{1}$ M. Hennessy, ${ }^{1}{ }^{\circ}$ M. Baldin, ${ }^{2} \odot$ and K. J. Harvatine ${ }^{3} \odot$ \\ ${ }^{1}$ Department of Clinical Studies, School of Veterinary Medicine, University of Pennsylvania, Kennett Square 19348 \\ ${ }^{2}$ MILC Group, San Luis Obispo, CA 93405 \\ ${ }^{3}$ Department of Animal Science, The Pennsylvania State University, University Park 16802
}

\begin{abstract}
Diet-induced milk fat depression (MFD) is a condition marked by a reduction in milk fat yield experimentally achieved by increasing dietary unsaturated fatty acids and fermentable carbohydrates. 2-Hydroxy4-(methylthio) butanoate (HMTBa) is a methionine analog observed to reduce diet-induced MFD in dairy cows. We hypothesize that the reduction in diet-induced MFD by HMTBa is due to changes in the rumen microbiota. To test this, 22 high-producing cannulated Holstein dairy cows were placed into 2 groups using a randomized block design and assigned to either control or HMTBa supplementation ( $0.1 \%$ of diet dry matter). All cows were then exposed to 3 different diets with a low risk (32\% neutral detergent fiber, no added oil; fed d 1 to 7 ), a moderate risk ( $29 \%$ neutral detergent fiber and $0.75 \%$ soybean oil; fed d 8 to 24 ), or a high risk (29\% neutral detergent fiber and $1.5 \%$ soybean oil; fed d 25 to 28) for diet-induced MFD. Rumen samples were collected on d 0, 14, 24, and 28, extracted for DNA, PCR-amplified for the V1-V2 region of the 16S rRNA gene, sequenced on an Illumina MiSeq (Illumina, San Diego, CA), and subjected to bacterial diversity analysis using the QIIME pipeline. The $\alpha$ diversity estimates (species richness and Shannon diversity) were decreased in the control group compared with the HMTBa group. Bacterial community composition also differed between control and HMTBa groups based on both weighted UniFrac (relative abundance of commonly detected bacteria) and unweighted UniFrac (presence/absence) distances. Within the HMTBa group, no differences were observed in bacterial community composition between $\mathrm{d} 0$ and d 14, 24, and 28; however, in the control group, d 0 samples were different from d 14, 24, and 28. Certain bacterial genera including Dialister,
\end{abstract}

Received August 2, 2019.

Accepted November 1, 2019.

*Corresponding author: dpitta@vet.upenn.edu
Megasphaera, Lachnospira, and Sharpea were increased in the control group compared with the HMTBa group. Interestingly, these genera were positively correlated with milk fat trans-10,cis-12 conjugated linoleic acid and trans-10 C18:1, fatty acid isomers associated with biohydrogenation-induced MFD. It can be concluded that diet-induced MFD is accompanied by significant alterations in the rumen bacterial community and that HMTBa supplementation reduces these microbial perturbations.

Key words: biohydrogenation, correlation, milk fat depression, milk fatty acid isomer

\section{INTRODUCTION}

Milk fat concentration is a major determinant of the economic and nutritional value of milk and is sensitive to external stimuli, particularly components of a dairy cow's diet. Milk fat concentration below the genetic potential of the cow when feeding diets that alter rumen fermentation is referred to as diet-induced or biohydrogenation (BH)-induced milk fat depression (MFD), a condition that does not influence milk yield or other milk components, but reduces the value of milk (Harvatine, 2016).

To better understand MFD, an experimental dietary model of lowering NDF and increasing starch and UFA was established to produce diet-induced MFD in dairy cows (Bauman et al., 1999; Rico and Harvatine, 2013; Rico et al., 2014). The decrease in milk fat yield during MFD is caused by bioactive fatty acids (FA) produced in the rumen (Bauman and Griinari, 2003). In ruminants, dietary PUFA are converted to saturated fats by rumen microbes through BH. During diet-induced MFD, $\mathrm{BH}$ pathways are altered in the rumen resulting in the formation of specific trans FA that, upon reaching the mammary gland, reduce milk fat synthesis (Harvatine et al., 2009). The severity of MFD is dependent upon the rate and extent of $\mathrm{BH}$ and the specific intermediates formed, which in turn are influenced by dietary risk factors including the concentrations of PUFA and 
fermentable carbohydrates in the diet (Jenkins et al., 2008; Fuentes et al., 2009). Although MFD in dairy cows is the direct consequence of rumen microbial activity, the role played by microbes in MFD is not clear.

2-Hydroxy-4-(methylthio) butanoate (HMTBa), an analog of methionine, is a feed additive that has the potential to modify milk fat yield (Zanton et al., 2014). 2-Hydroxy-4-(methylthio) butanoate is available in the rumen, where it is rapidly absorbed and converted to methionine, thus having a methionine-sparing effect in ruminants (Lobley et al., 2006). Reports suggest that HMTBa may support fiber degradation and microbial protein synthesis and, ultimately, better fermentation in the rumen (Lee et al., 2015). 2-Hydroxy-4-(methylthio) butanoate may interact with cellulolytic bacteria directly and may indirectly influence non-cellulolytic bacteria in the rumen (Martin et al., 2013). Although the production responses of HMTBa have been attributed to alteration of the ruminal environment, knowledge of the effects of HMTBa on ruminal microbiota is sparse.

Decreasing forage NDF and increasing starch and UFA are dietary factors well described to increase risk of diet-induced MFD as they lead to a switch to altered $\mathrm{BH}$ pathways in the rumen, resulting in the formation of bioactive FA that cause MFD. Baldin et al. (2018) and M. Baldin, G. I. Zanton (USDA, Madison, WI), and K. J. Harvatine (unpublished data) have used these dietary factors to expose dairy cows to low, moderate, and high levels of risk for MFD and have evaluated the effects of HMTBa supplementation on alleviating the condition. These authors found that HMTBa supplementation was effective in maintaining milk fat yield and concentration during moderate- and high-risk diet scenarios. Furthermore, they found that HMTBa supplementation maintained lower concentrations of the trans-10 C18:1 isomer in milk, indicating that the shift to altered BH in the rumen is inhibited by HMTBa supplementation. The current study was performed as an accompaniment to M. Baldin, G. I. Zanton (USDA, Madison, WI), and K. J. Harvatine (unpublished data) based on the hypothesis that HMTBa-mediated inhibition of BH-induced MFD is modulated by alterations in the ruminal microbiota. To this end, we analyzed changes in ruminal bacterial populations and their associations with milk FA in dairy cows exposed to dietary induction of MFD with and without HMTBa supplementation.

\section{MATERIALS AND METHODS}

\section{Experimental Design and Treatments}

The design of the experiment and animal assignment to treatments were previously described by $\mathrm{M}$.
Baldin, G. I. Zanton (USDA, Madison, WI), and K. J. Harvatine (unpublished data) and were approved by the Pennsylvania State University Institutional Animal Care and Use Committee. Briefly, 22 ruminally cannulated multiparous Holstein cows were used in a randomized block design. All cows were maintained on a diet with low risk for MFD during a 7-d pretrial period. At the end of the pretrial period, cows were paired by milk production and were randomly assigned to 2 treatments: unsupplemented control or HMTBa supplemented group. Details of the experimental diets are presented (Supplemental Table S1; https://doi.org/ 10.3168/jds.2019-17389). The HMTBa (Alimet; Novus International Inc., St. Charles, MO) was provided at $0.1 \%$ of dietary DM in a corn carrier (10\% HMTBa) and mixed into the TMR. The control group received an equal amount of the same ground corn carrier in their diets. During the course of the experimental period, animals were fed a diet with low risk of $\mathrm{BH}-$ induced MFD from d 1 to 7 (31.7\% NDF, $27.2 \%$ starch, $2.18 \%$ UFA, no added oil), moderate risk from d 8 to 24 (29.1\% NDF, $29.3 \%$ starch, 3.80\% UFA), and high risk from d 25 to 28 (28.7\% NDF, $29.6 \%$ starch, $4.45 \%$ UFA). Dietary UFA were increased using a combination of rapidly available FA from soybean oil and more slowly available FA from roasted soybeans. Diets were fed during pretrial and treatment periods as a TMR once daily at $0700 \mathrm{~h}$ at $110 \%$ of expected daily intake.

\section{Sampling and Measurements}

Procedures for feed intake and milk responses were previously described by M. Baldin, G. I. Zanton (USDA, Madison, WI), and K. J. Harvatine (unpublished data). Feed intake was measured daily. Feed ingredients were sampled once per week and stored at $-20^{\circ} \mathrm{C}$, thawed at room temperature, dried at $55^{\circ} \mathrm{C}$ in a forced-air oven for $72 \mathrm{~h}$, and ground in a Wiley mill through a 1-mm screen (A. H. Thomas, Philadelphia, PA). Feed samples were composited within dietary phase (equal dry weight basis). Feed samples were analyzed for CP, NDF, and ADF by wet chemistry procedures (Cumberland Valley Analytical Services Inc., Maugansville, MD). Briefly, $\mathrm{CP}$ was determined according to AOAC International (2000) using method 990.03, ADF was determined according to AOAC International (2000) method 973.18, and ash-free NDF according to Van Soest et al. (1991) using heat-stable amylase and sodium sulfite. Starch was determined by an enzymatic method (Karkalas, 1985; Hazyme, Centerchem, Norwalk, CT) after samples were gelatinized with sodium hydroxide. Total FA concentration and FA profile of feed samples were determined by GC after direct methylation (Sukhija and Palmquist, 1988), as described by Rico and Harvatine (2013). 
Cows were milked twice daily at 0600 and $1800 \mathrm{~h}$ and milk yield was determined by an integrated milk meter (AfiMilk, SAE Afikim, Afikim, Israel). Milk was sampled at both milkings on d $0,7,14,24$, and 28, and was composited based on yield at each milking. One milk sample from the composite was stored at $4^{\circ} \mathrm{C}$ with preservative (Bronolab-WII, Advanced Instruments Inc., Norwood, MA) until analysis for fat and protein content by Fourier transform infrared spectroscopy (Fossomatic 4000 Milko-Scan and 400 Fossomatic, Foss Electric, Hillerød, Denmark; at Dairy One Laboratory, Ithaca, NY). A second milk sample was immediately centrifuged at $3,000 \times g$ for $15 \mathrm{~min}$ at $4^{\circ} \mathrm{C}$ and the fat cake was stored at $-20^{\circ} \mathrm{C}$ before analysis of FA composition, as described by Baldin et al. (2018). Briefly, FA were extracted in hexane isopropanol, base transmethylated with sodium methoxide, and quantified by GC with a fused-silica capillary column (SP-2560, $100 \mathrm{~m} \times$ $0.25 \mathrm{~mm}$ i.d. with $0.2-\mu \mathrm{m}$ film thickness; Supelco Inc., Bellefonte, PA) and a flame ionization detector.

Whole rumen digesta was collected via cannula twice daily (approximately $1 \mathrm{~h}$ before and $6 \mathrm{~h}$ after feeding) on d $0,7,14,24$, and 28. Samples were collected from 5 different locations in the rumen (cranial dorsal, cranial ventral, central, caudal dorsal, and caudal ventral), mixed in a bucket, and a composite subsample of approximately $200 \mathrm{~g}$ was immediately placed on dry ice and archived at $-80^{\circ} \mathrm{C}$ until analysis of microbial populations was performed.

\section{Genomic DNA Extraction and PCR Amplification}

The genomic DNA from whole digesta samples was extracted using bead-beating followed by precipitation with isopropanol and ethanol and finally extraction with a commercial kit (QIAamp DNA Stool Mini Kit; Qiagen Sciences, Germantown, MD) based on the procedure described in $\mathrm{Yu}$ and Morrison (2004). For each extracted genomic DNA sample, the V1-V2 region of bacterial $16 \mathrm{~S}$ rRNA gene was PCR-amplified in triplicate using the bacterial-specific primers F27 (5'-GAGTTTGATCCTGGCTCAG-3') and R388 (5'-TGCTGCCTCCCGTAGGAGT-3') barcoded with a unique 12-base error-correcting Golay code for multiplexing as described in Song et al. (2013). Polymerase chain reaction was performed using the Accuprime Taq DNA Polymerase System (Invitrogen, Carlsbad, $\mathrm{CA})$. The thermal cycling conditions involved an initial denaturing step at $95^{\circ} \mathrm{C}$ for 5 min followed by 20 cycles (denaturing at $95^{\circ} \mathrm{C}$ for $30 \mathrm{~s}$, annealing at $56^{\circ} \mathrm{C}$ for $30 \mathrm{~s}$, extension at $72^{\circ} \mathrm{C}$ for $90 \mathrm{~s}$ ) and an extension step at $72^{\circ} \mathrm{C}$ for $8 \mathrm{~min}$. The amplicons from each DNA sample were combined and then each library was added to a pool in equimolar concentration. The final pool was bead purified using Beckman Coulter Agencourt AMPure XP Beads (Beckman Coulter, Brea, CA). Multiplex DNA sequencing was performed with an Illumina MiSeq (Illumina, San Diego, CA) to obtain $2 \times$ 250 bp paired-end reads. Raw sequencing information was deposited at National Center for Biotechnological Information within the Short Read Archive under the project accession number PRJNA580199.

\section{Sequencing, Data Analysis, and Statistical Analysis}

The 16S rDNA reads were analyzed using QIIME 1.8.0 pipeline (Caporaso et al., 2010b). First, the forward and reverse Illumina reads were joined together using the join_paired_ends.py script. The merged sequences were de-multiplexed and quality filtered. The operational taxonomic units (OTU) were formed by clustering sequences based on 97\% similarity threshold using the UCLUST algorithm (Edgar, 2010). Representative sequences for each OTU were aligned with PyNast (Caporaso et al., 2010a). The resultant multiple sequence alignment was used to infer a phylogenetic tree with FastTree (Price et al., 2010). The taxonomy of each sequence was identified using uclust consensus taxonomy assigner by performing a search against GreenGenes taxonomy (12/10 release; McDonald et al., 2012). The $16 \mathrm{~S}$ sequence information from the before and after feeding samples was combined to form one representative sample. The OTU tables were rarefied at 48,536 sequences per sample for $\alpha$ and $\beta$ diversity analysis. Package qiimer (Bittinger, 2015) was used to open QIIME output files in R (version 3.1.1; $\mathrm{R}$ Core Team, 2019). Analyses of community similarity ( $\beta$ diversity) were performed by calculating pairwise distance using phylogenetic metric UniFrac. Two measures of $\alpha$ diversity were computed including Shannon diversity, an indicator of evenness in community structure, and richness, the number of OTU observed. The measured $\alpha$ diversity matrices were compared between the treatment groups using Wilcoxon rank sum test. A nonparametric permutational multivariate ANOVA test (Anderson, 2001), implemented in the vegan package for $\mathrm{R}$, was used to test the effects of day of sampling and treatment within the day of sampling on overall community composition, as measured by weighted and unweighted UniFrac distance. The raw read counts from the $16 \mathrm{~S}$ rDNA OTU abundance table were collapsed at taxonomic rank and compositionally normalized (relative abundance) such that each sample sums to 1 . To test the differences in bacterial taxa between control and HMTBa treatment groups across all days of sampling, analysis of composition of microbiomes (ANCOM; Mandal et al., 2015) available in $\mathrm{R}$ was used. The significance of test is determined 
using the Benjamini-Hochberg procedure that controls for false discovery rate. Further, the taxa that differed significantly between the 2 groups using the ANCOM test were tested for significance at each day of sampling using Wilcoxon rank sum test. A $P$-value of 0.05 was used to define significance. The Spearman correlation coefficients $\left(r_{s}\right)$ were calculated to evaluate correlations between genera and FA profiles.

\section{RESULTS}

\section{Milk Production Responses}

In the accompanying study by M. Baldin, G. I. Zanton (USDA, Madison, WI), and K. J. Harvatine (unpublished data), feeding a moderate-risk diet was found to be sufficient to cause diet-induced MFD, as evidenced by a 2.5 -fold increase in trans-10 C18:1 and a 13\% reduction in milk fat concentration in the control group between d 7 (end of low risk diet) and d 24 (end of moderate-risk diet; $P<0.01$ for both variables). Milk fat yield was significantly higher in the HMTBa group at d 14 (midpoint of moderate diet; $P<0.05$ ), d $24(P<0.05)$, and d 28 (end of high-risk diet; $P$ $<0.05$ ) as compared with the control (Supplemental Figure S1; https://doi.org/10.3168/jds.2019-17389). In addition, trans-10 C18:1 was significantly lower in the HMTBa group at $\mathrm{d} 14,24$, and $28(P<0.05$ for all time points), indicating that HMTBa was successful in preventing the shift to the altered $\mathrm{BH}$ pathway. No significant change was seen between the 2 groups in trans-11 C18:1.

\section{Sequencing Details}

According to M. Baldin, G. I. Zanton (USDA, Madison, WI), and K. J. Harvatine (unpublished data), no significant differences were observed between control and HMTBa on the low-risk diet (d 7), and for this reason, the rumen samples from d 7 were not analyzed for bacterial information in this study. Almost $15416 \mathrm{~S}$ rRNA-based bacterial communities that contributed to approximately 1 million high quality reads were analyzed. These reads were binned into 137,563 OTU (minimum: 48,536; maximum: 103,131; mean: 72,392). The range for Good's coverage across all samples was 94 to $97 \%$, indicating a good sequencing depth per sample.

\section{Within-Sample Variation (Alpha Diversity)}

The number of bacterial species (OTU) was estimated by species richness and the distribution of bacterial species within each community was estimated by Shannon diversity index (Figure 1a-f; Supplemental Table S2; https://doi.org/10.3168/jds.2019-17389). Both species richness (Figure 1a) and Shannon diversity (Figure 1d) were reduced $(P<0.05)$ in the control compared with HMTBa across all days with increasing risk of BHinduced MFD. No significant differences were noted between d 0 and d 14, 24, and 28 for observed species in either control or HMTBa (Figure 1b, c). However, within the control, compared with d 0, d 14, 24, and 28 showed greater variation with the most noticeable variation on d 28 during the highest risk phase (Figure $1 b$ ), whereas no such variation was noted between days of sampling with HMTBa (Figure 1c). For Shannon diversity, differences were noted between d 0 compared with d 14, 24, and 28 in the control group only (Figure 1e). Interestingly, no differences were noted between $\mathrm{d}$ 14, 24, and 28 in either control or HMTBa (Supplemental Table S2).

\section{Between-Bacterial Community Comparison (Beta Diversity)}

The weighted and unweighted UniFrac distances were used to derive principal coordinates (Figure $2 \mathrm{a}$ and $2 \mathrm{~b}$ ). Bacterial communities differed $(P<0.05$; Permanova test; Table 1) by dietary risk of MFD and treatment in both weighted (relative abundance of commonly present bacterial taxa) and unweighted (presence-absence information of bacterial taxa) distance matrices. In both weighted and unweighted matrices, in the control, bacterial community composition differed $(P<0.05)$ on d 0 as compared with d 14, 24, and 28, whereas no such differences were noted in the HMTBa group. In both control and HMTBa, bacterial communities did not differ $(P>0.05)$ between d 14 and $\mathrm{d} 24$ and 28 (moderate and high risk of MFD).

\section{Phylogenetic Composition of Bacterial Communities in the Rumen}

Representative sequences from the OTU information were assigned to 21 bacterial phyla and 351 genera; however, only 83 genera were present across $75 \%$ of the samples (Supplemental Table S3; https://doi.org/ 10.3168/jds.2019-17389). The most dominant bacterial phyla were Firmicutes (47.84\%) and Bacteroidetes $(41.46 \%)$, which together constituted approximately $90 \%$ of total bacterial abundance. The other less abundant phyla included Proteobacteria $(3.10 \%)$, Fibrobacteres (2.90\%), Spirochaetes (1.83\%), Cyanobacteria $(0.77 \%)$, Tenericutes $(0.77 \%)$, and Actinobacteria (0.52\%). Among Firmicutes, Lachnospiraceae (10.72\%), Clostridiales (10.22\%), Butyrivibrio (6.25\%), Rumino- 
coccaceae (6.06\%), Ruminococcus (5.77\%), Coprococcus (3.55\%), and Succiniclasticum (3.38\%) were the most abundant bacterial lineages. Bacteroidetes was dominated by Prevotella (34.56\%), Bacteroidales (5.96\%), S24-7 (3.58\%), Paraprevotellaceae (0.87\%), and RF16 (0.86\%). Proteobacteria was dominated by Succinivibrionaceae (4.13\%), Fibrobacteres by Fibrobacter (2.90\%), Spirochaetes by Treponema (3.34\%), Cyanobacteria by YS2 (2.17\%), Tenericutes by Anaeroplasma (1.99\%) and RF39 (1.97\%), and Actinobacteria by Coriobacteriaceae $(2.08 \%)$ members.

\section{Changes in Ruminal Bacteria with MFD Dietary Risks and Effects of HMTBa}

To compare changes in bacterial populations between HMTBa and control, a new tool, the ANCOM test (Mandal et al., 2015), was used; this tool was selected because it includes rare bacterial populations. Using this tool, 7 genera were identified that differed between control and HMTBa across all days of sampling (Figure $3 ; P<0.05)$. The d 0 sample was used as a covariate. The ANCOM analysis revealed an overall increase $(P<$

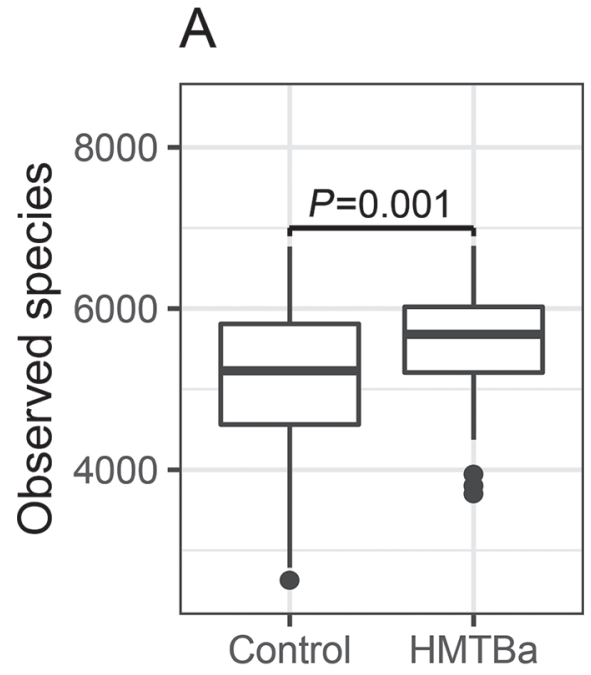

D

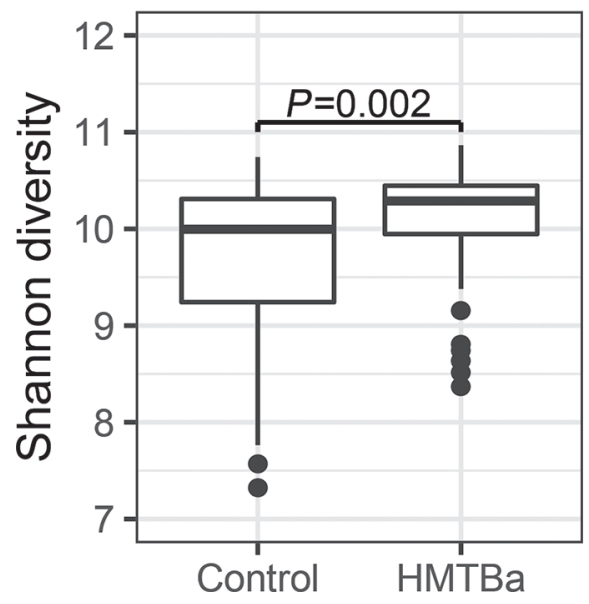

B: Control

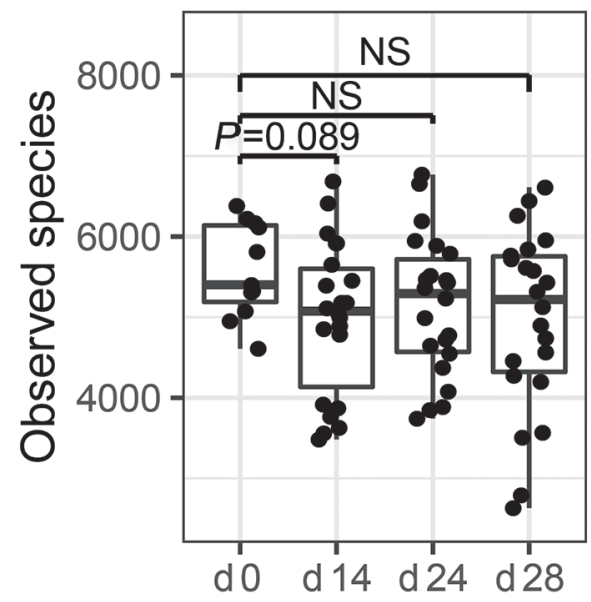

E: Control

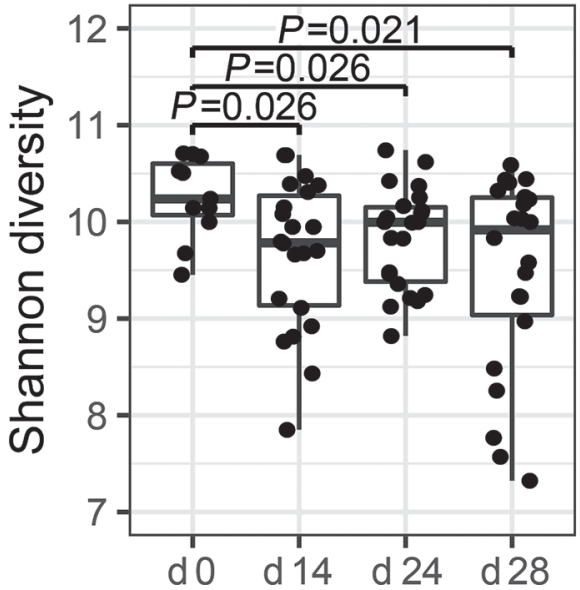

C: HMTBa

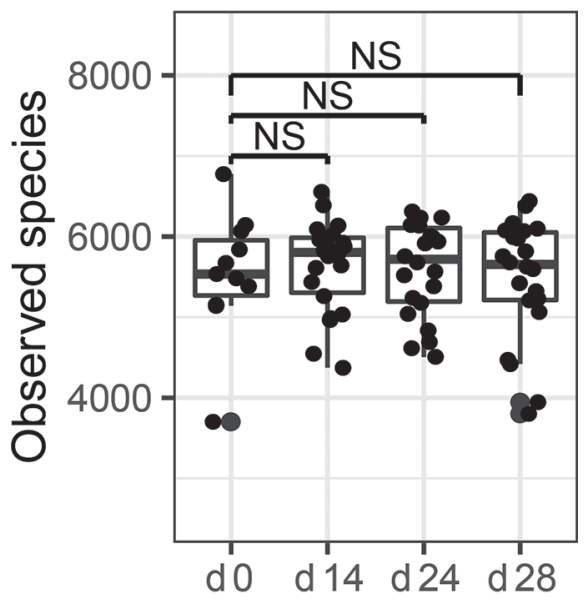

Figure 1. Measurement of community diversity ( $\alpha$ diversity) of the rumen bacterial communities fed control or 2-hydroxy-4-(methylthio) butanoate $(\mathrm{HMTBa})$ in diets with increasing risk of biohydrogenation-induced milk fat depression $(\mathrm{d} 0=$ covariant, $\mathrm{d} 14=$ moderate risk, $\mathrm{d} 24$ $=$ moderate risk, and d $28=$ high risk). Panels include (A) number of observed species between treatment groups, (B) number of observed species between days in the control, (C) number of observed species between days in the HMTBa treatment, (D) Shannon diversity between treatments, (E) Shannon diversity between days in the control, and (F) Shannon diversity between days in the HMTBa treatment. Boxes represent the interquartile range (IQR), and the lines inside represent the median. Whiskers denote the lowest and highest values within 1.5 times of the $\mathrm{IQR}$; dots denote the diversity index observations for each sample. 
Table 1. Permutational multivariate ANOVA for the effect of 2-hydroxy-4-(methylthio) butanoate (HMTBa) and day of sampling while feeding diets with increasing risk of biohydrogenation-induced milk fat depression $(\mathrm{d} 0=$ covariant, $\mathrm{d} 14=$ low risk, $\mathrm{d} 24=$ moderate risk, and d $28=$ high risk) on ruminal bacterial community composition based on weighted and unweighted UniFrac distances

\begin{tabular}{lcc}
\hline Item & Weighted & Unweighted \\
\hline Treatment & 0.01 & 0.001 \\
Day & 0.003 & 0.002 \\
Treatment $\times$ day & 0.482 & 0.458 \\
Control group & 0.021 & 0.028 \\
Day (overall) & 0.045 & 0.02 \\
d 0 vs. d 14 & 0.016 & 0.022 \\
d 0 vs. d 21 & 0.013 & 0.011 \\
d 0 vs. d 28 & 0.089 & 0.176 \\
d 14 vs. d 24 & 0.295 & 0.261 \\
d 14 vs. d 28 & 0.123 & 0.269 \\
d 24 vs. d 28 & & \\
HMTBa group & 0.128 & 0.118 \\
Day (overall) & 0.136 & 0.143 \\
d 0 vs. d 14 & 0.064 & 0.076 \\
d 0 vs. d 21 & 0.128 & 0.079 \\
d 0 vs. d 28 & 0.349 & 0.693 \\
d 14 vs. d 24 & 0.135 & 0.182 \\
d 14 vs. d 28 & 0.349 & 0.388 \\
d 24 vs. d 28 & &
\end{tabular}

0.05) in the relative abundance of Dialister, Lachnospira, Megasphaera, and Sharpea in the control compared with HMTBa. Within the control group, these 4 genera were increased on d 14 (moderate-risk phase) compared with d 0. The genera Dialister and Megasphaera were reduced on d 24 (moderate-risk phase) compared with d 14 but increased on d 28 (high risk phase) compared with d 24 in the control. In HMTBa, these 2 genera were reduced on d 14, 24, and 28 compared with d 0 .
However, the magnitude of decrease was greater on $\mathrm{d}$ 14 and 24 compared with d 28. A different pattern was noted for the genera Lachnospira and Sharpea in the control group, where these 2 genera were increased on $\mathrm{d}$ 14 and then gradually reduced by d 28 . In the HMTBa group, the relative abundance of these 2 genera was sustained from d 0 to 28 . In contrast, SR1, L7A_E11 from Firmicutes, and F16 from TM7 were reduced in the control group compared with the HMTBa group $(P<0.05)$. Both $L 7 A \_E 11$ and $F 16$ were reduced on d 14 compared with d 0 and remained low through d 24 and 28 (moderate- and high-risk phases). Genus SR1 was gradually decreased from d 0 to 28 in HMTBa but remained higher compared with the control.

\section{Correlation Analysis Between Bacteria Associated with MFD Dietary Risks and Milk FA Profiles}

Associations between bacterial taxa and production responses were analyzed using Spearman correlation (Supplemental Table S4; https://doi.org/10.3168/jds .2019-17389). Bacterial genera that were significantly positively or negatively correlated with a coefficient value of $>0.3$ were considered (Figure 4). Coefficient values $>0.5$ were considered strong, whereas those between 0.3 and 0.5 were considered weak. The analysis showed that very few bacterial populations at the genus level had positive correlations with DMI and milk yield, with the notable exceptions of Coriobacteriaceae, Dialister, and Veillonellaceae. It is evident that most bacterial lineages from Firmicutes were positively correlated with milk fat yield and concentration. The representa-
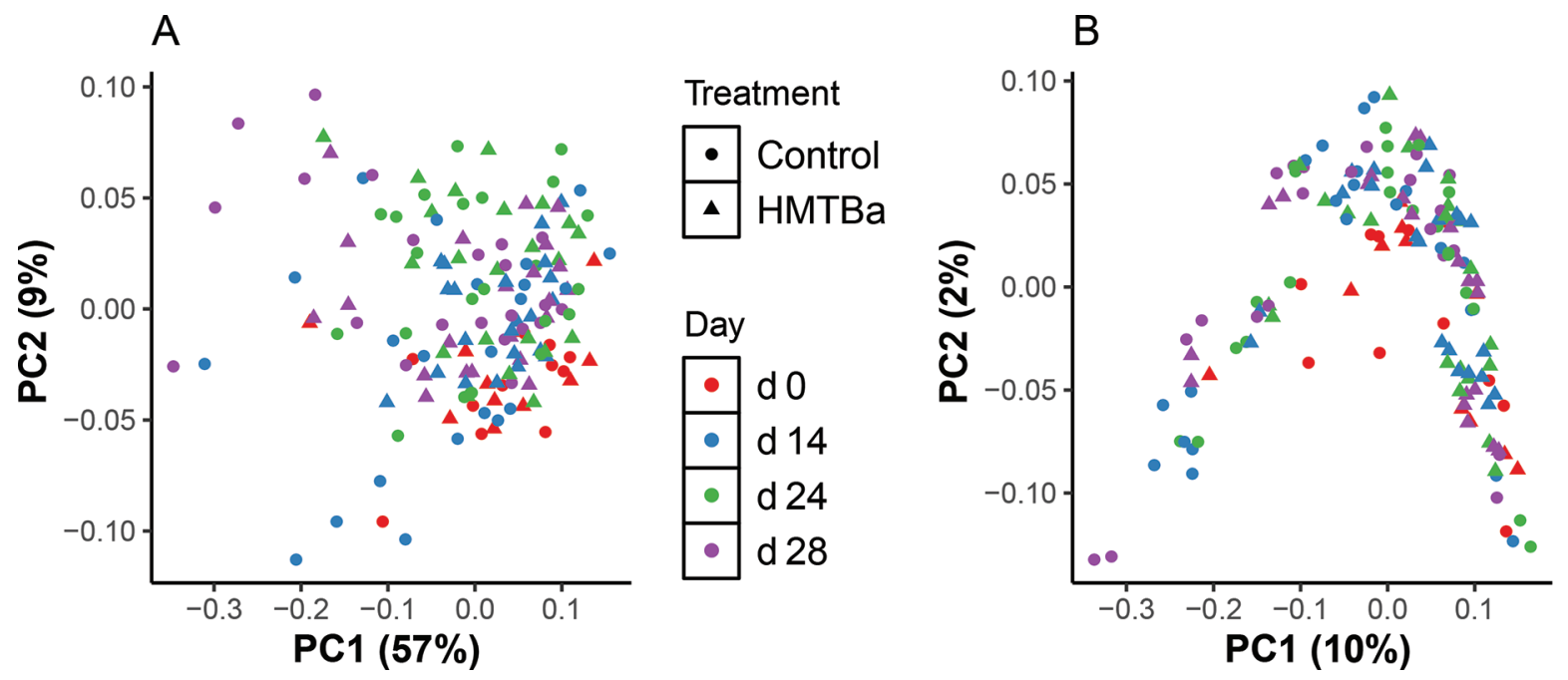
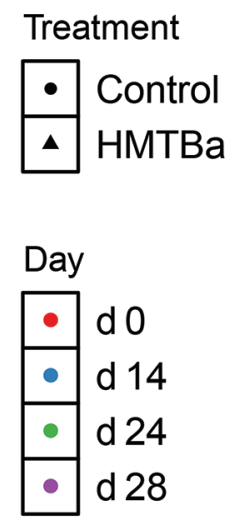

Figure 2. Comparison of bacterial community composition between control and 2-hydroxy-4-(methylthio) butanoate (HMTBa) supplemen-

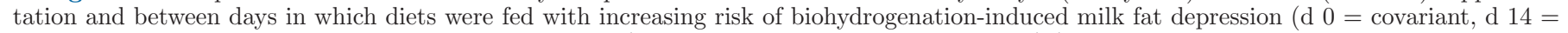

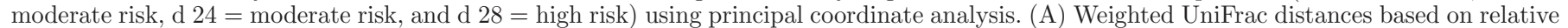

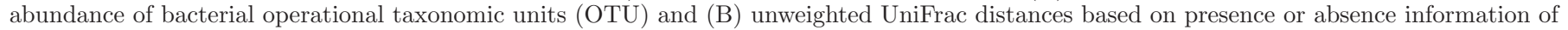
bacterial OTU $(\mathrm{PC} 1=$ principal coordinate $1 ; \mathrm{PC} 2=$ principal coordinate 2$)$. 
tives from this phylum, including Blautia, Anaerovibrio, Selenomonas, Christenellaceae, and Clostridiales, showed a high positive correlation $(P<0.05)$ with milk fat concentration and a weak positive correlation $(P<$ $0.05)$ with milk fat yield. None of the bacterial populations had strong positive correlations with milk protein percentage, whereas a few populations, including $D i$ alister, Eubacterium, and Acidaminococcus, had weak negative correlations. No interesting patterns were noted between protein yield and bacterial populations.
For the FA isomers, very few bacterial genera showed a weak positive correlation with cis-9,trans-11 CLA, including Dialister and Sharpea, whereas BS11, Christensenellaceae, and Paludibacter had a negative correlation with this isomer. None of the bacterial populations had either strong positive or negative associations with trans-11 C18:1, the main intermediate of the normal BH pathway. The bacterial genera that had a significant $(P<0.05)$ positive correlation to both trans-10, cis-12 CLA and trans-10 C18:1 isomers were Coriobacteriace-
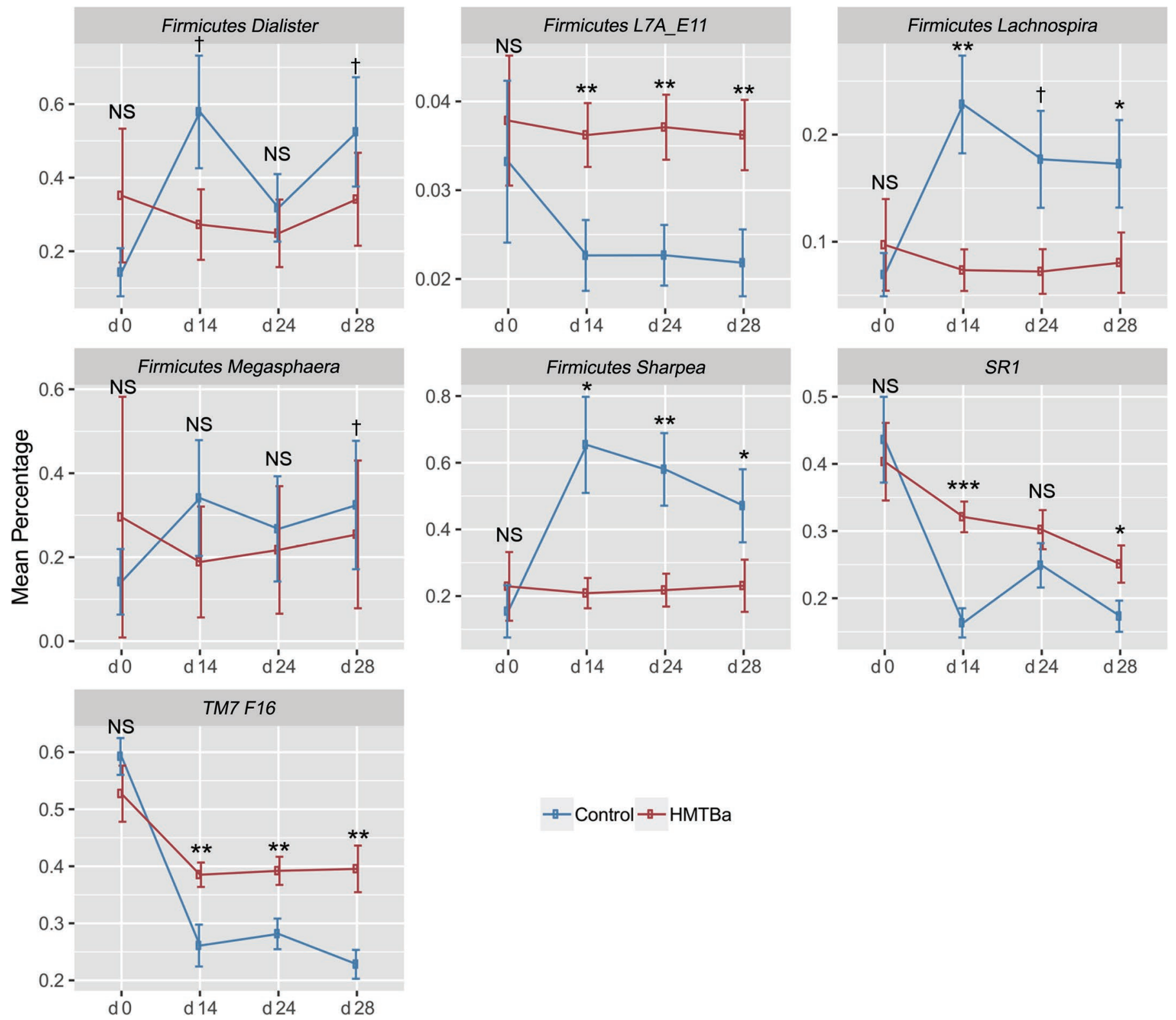

Figure 3. Significantly differentially abundant bacterial genera identified by analysis of composition of microbiomes (ANCOM; Mandal et al., 2015) between control and 2-hydroxy-4-(methylthio) butanoate (HMTBa) supplementation and between days in which diets were fed with increasing risk of biohydrogenation-induced milk fat depression $(\mathrm{d} 0=$ covariant, $\mathrm{d} 14=$ moderate risk, $\mathrm{d} 24=$ moderate risk, and $\mathrm{d} 28=$ high risk). The effect of HMTBa is identified within each day based on Wilcoxon rank sum test and shown on the plots $(* * * P<0.001 ; * * P<0.01$; $* P<0.05 ; \dagger P<0.10)$. The error bars indicate SEM. 

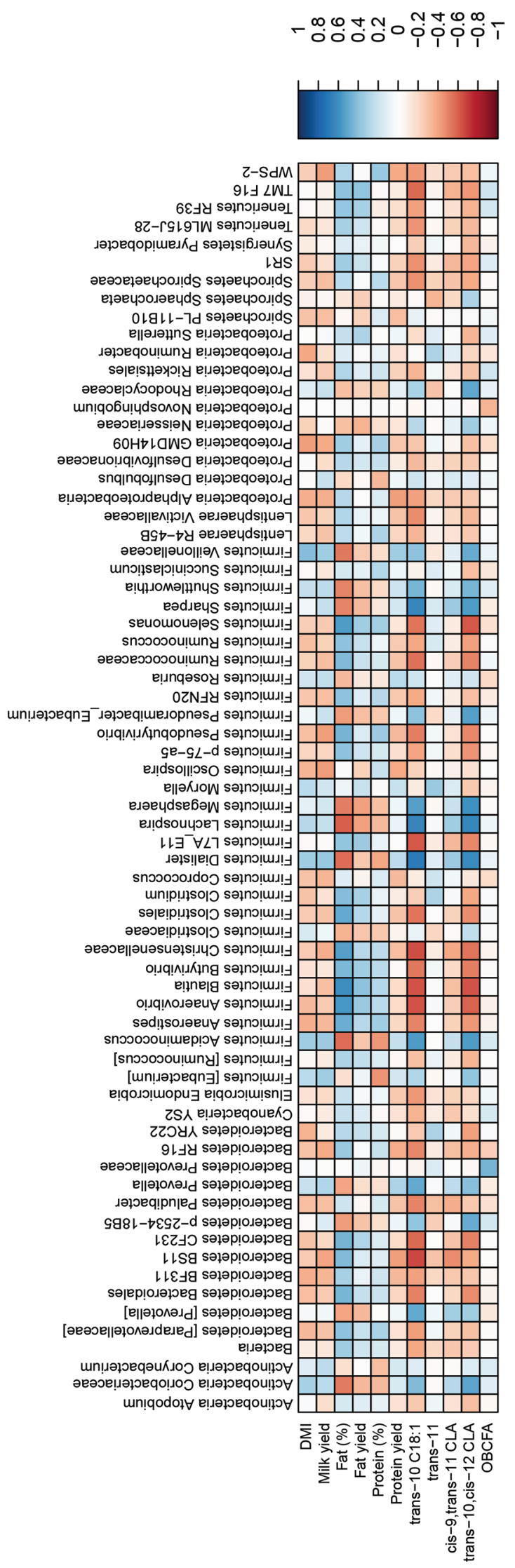

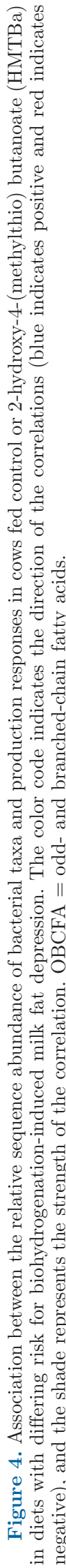

ae, Prevotella, Acidaminococcus, Dialister, Lachnospira, Megasphaera, Pseudoramibacter-Eubacterium, Sharpea, Shuttleworthia, and Veillonellaceae. Interestingly, Dialister, Lachnospira, Megasphaera, and Sharpea, all of which increased in the control, were positively correlated $(P<0.05)$ not only with the 2 trans-10 isomers of the alternate $\mathrm{BH}$ pathway, but also with milk yield. These genera were negatively correlated $(P<0.05)$ with milk fat and protein concentration.

\section{DISCUSSION}

\section{Dietary Models to Investigate MFD}

Diet-induced MFD continues to be a common problem in dairy herds despite improved nutritional management (Baldin et al., 2017). Several factors, including the concentrations and availability of FA in dairy cow rations, fermentability of diets, feeding management, rumen modifiers, and individual cow-to-cow factors, predispose dairy cows to MFD (Harvatine, 2016). Experimental induction and recovery models have been useful in investigation of the pathophysiology of diet-induced MFD (Rico and Harvatine, 2013; Rico et al., 2014). Briefly, in this model, MFD is consistently achieved within $10 \mathrm{~d}$ by reducing forage NDF and increasing both starch and PUFA and rescued within 10 to $18 \mathrm{~d}$ of returning to a recovery diet. Increasing levels of dietary starch are known to reduce ruminal $\mathrm{pH}$ and consequently alter microbial populations (Fuentes et al., 2009), while increasing levels of dietary PUFA are known to inhibit the growth of microbial populations (Maia et al., 2010). Because these dietary modifications significantly alter the ruminal environment, including the microbiota, they lead to a progressive buildup of trans-10, cis-12 CLA and trans-10 isomers in the rumen, which are associated with diet-induced MFD (Rico et al., 2014, 2015). Using an induction-recovery model of MFD, Rico et al. (2015) and Pitta et al. (2018) have previously demonstrated significant alterations in the ruminal microbial populations by d 10 of the induction phase and restoration to the original state by $\mathrm{d} 14$ of the recovery phase. Collectively, these studies established the link between dietary risk factors, rumen microbial composition, and milk FA and provided insights into the physiology behind diet-induced MFD.

A challenge model has been useful in testing the effect of feed or supplements on induction of diet-induced MFD. Baldin et al. (2018, 2019), and M. Baldin, G. I. Zanton (USDA, Madison, WI), and K. J. Harvatine (unpublished data) investigated the effect of HMTBa on risk for MFD in 3 separate experiments with designs that sequentially increased dietary MFD risk factors to represent low-, moderate-, and high-risk diets. This 
sequential increase in dietary MFD risk allows the evaluation of the effects of HMTBa in preventing the shift to altered $\mathrm{BH}$ while reducing the need for washout periods. The experiments differed in the degree of challenge and length of feeding challenge diets. Similar to previous investigation of the effect of HMTBa in the challenge model, HMTBa had no effect on the low risk diet, but reduced the shift to trans-10 pathway and induction of MFD on the moderate- and high-risk phases [M. Baldin, G. I. Zanton (USDA, Madison, WI), and K. J. Harvatine, unpublished data].

\section{Changes in Ruminal Microbiota with Increasing Dietary MFD Risks}

Because M. Baldin, G. I. Zanton (USDA, Madison, WI), and K. J. Harvatine (unpublished data) found no significant change in FA isomers between control and HMTBa groups at d 7 (low-risk phase), those ruminal samples were not analyzed in the current study with the presumption that microbial populations are acclimating to the low-risk diet and it may be too early to find differences in bacterial communities at this stage. However, the bacterial community composition in ruminal samples from dairy cows on moderate-risk (d 14 and 24) and high-risk (d 28) MFD diets were found to be different from those in $\mathrm{d} 0$ samples in the control group, indicating that MFD dietary risks induce changes in ruminal bacterial communities. This finding is similar to our previous report (Pitta et al., 2018), which found that the MFD induction diet is associated with altered bacterial communities when compared with recovered bacterial communities. The moderateand high-risk diets had the same concentration of NDF and starch, but the high-risk diet had a higher PUFA concentration. Interestingly, no differences in the bacterial community composition between moderate- and high-risk diets were identified in this study, indicating that both fermentable carbohydrates and PUFA concentrations drive alterations in ruminal bacteria. However, the high-risk period was maintained for only $5 \mathrm{~d}$, and further studies may be required to investigate the effect of further increasing PUFA concentrations alone for longer periods $(>5 \mathrm{~d}$ ) on ruminal bacteria.

During the course of the MFD induction period, Rico et al. (2014) reported a gradual reduction of cis-9,trans-11 CLA isomer and a progressive buildup of trans-10,cis-12 CLA isomer. Pitta et al. (2018), in an accompanied study to Rico et al. (2014), identified that members of Firmicutes and Actinobacteria were increased whereas members of Bacteroidetes were decreased at d 10 of the induction period. In the current study, Firmicutes remained stable whereas Actinobacteria doubled in both moderate and high MFD dietary risk samples compared with d 0 . It is possible that differences in NDF concentrations [26.1\% in the induction diet in Pitta et al., 2018 and Rico et al., 2014 vs. 29.1 and $28.7 \%$ in moderate- and high-risk diets in the current study and M. Baldin, G. I. Zanton (USDA, Madison, WI), and K. J. Harvatine (unpublished data)] may have resulted in differences in the relative abundance of Firmicutes between the 2 studies. Increased Actinobacteria, represented mostly by Coriobacteriaceae, as observed in this study, has been associated with dyslipidemic phenotypes (Martinez et al., 2013) and is known to produce trans-10 isomer (Zened et al., 2011).

In this study, certain bacterial genera such as $D i$ alister, Lachnospira, Megasphaera, and Sharpea were elevated on both moderate- and high-risk MFD diets and were positively correlated $(P<0.05)$ with trans10,cis-12 CLA and trans-10 isomers. These findings corroborate with Dewanckele et al. (2018), where these 4 genera, along with Bifidobacterium, Coriobacteriaceae, Lactobacillus, and Eubacterium, were positively associated with trans-10 isomers. It has also been previously shown (Latham et al., 1972) that 4 lactating dairy cows, when fed a high-roughage diet $(8 \mathrm{~kg}$ of hay and $10 \mathrm{~kg}$ of concentrate) and gradually transitioned to a milk fatdepressing low-roughage diet (until milk fat \% reduced to $<2 \%$ ), had an increase in Megasphaera elsdenii, Lactobacillus, Bifidobacterium, and Lachnospira multiparus on milk fat-depressing diets, which is in agreement with findings in this study. An increase in the relative population size of $M$. elsdenii in cows with MFD has been previously reported (Palmonari et al., 2010; Weimer et al., 2010; Mohammed et al., 2012). While certain strains of M. elsdenii, such as YJ-4, have been shown to produce trans-10,cis-12 CLA isomer (Kim et al., 2002), several other strains of M. elsdenii were unable to produce the same isomer in in vitro models (Wallace et al., 2007; Maia et al., 2007). Although the specific species of Megasphaera was not identified in this study, the relative abundance of this genus increased in cows with diet-induced MFD. Further studies on identification of the specific bacteria involved in the production of trans-10,cis-12 CLA isomers will be useful to develop strategies for reducing MFD in dairy cows. Application of metagenomic and metatranscriptomic approaches to analyze rumen samples may help to identify the bacteria as well as describe their functional role involved in the production of isomers that lead to MFD.

Some uncultured bacterial lineages, such as those within Lachnospiraceae, have been reported to participate in ruminal BH (Huws et al., 2011), although the specific mechanism is not well understood. In our previous study (Pitta et al., 2018), we have reported the association of unclassified Lachnospiraceae with trans-10 isomer in the rumen of dairy cows with diet-induced 
MFD. Interestingly, under a similar dietary regimen as that used in this study, we found Lachnospira, a member of Lachnospiraceae, to be associated with trans-10 isomer. All 4 of the genera discussed above were increased on $\mathrm{d} 14$ after $7 \mathrm{~d}$ on the moderate-risk diet, but declined toward the end of d 24, suggesting a possible adaptation of the dairy cows' ruminal microbiota to the moderate-risk diet. Upon switching to a high-risk diet on d 24, Dialister and Megasphaera increased by d 28 , suggesting these genera may have a role in the production of trans-10 isomers; however, such observations require further investigations to demonstrate the causeeffect relationship. The other 2 genera, Lachnospira and Sharpea, did not increase with higher UFA, suggesting that their increased abundance may be associated with changes in the ruminal environment induced by interactions between simultaneously increasing starch and fat concentrations. Kamke et al. (2016) reported that in sheep with lower methane-yield phenotype, a rapid heterofermentation is supported by an enrichment of Sharpea and Megasphaera spp. in the rumen, leading to lower hydrogen production, thus naturally controlling for methane formation. Collectively, these data support that rapid fermentation in the rumen induced by higher starch concentrations is conducive for specific bacterial phylotypes such as Sharpea, Megasphaera, and Lachnospiraceae. These changes in ruminal conditions, when paired with higher UFA as noted in this study, may lead to significant alterations in the rumen including shifting $\mathrm{BH}$ pathways.

\section{Effect of HMTBa on Reducing Perturbations in Ruminal Microbiota}

Several reports indicate that dairy cows supplemented with methionine hydroxy analogs have demonstrated improvements in bacterial protein synthesis (Arambel et al., 1987), protozoal counts (Lundquist et al., 1985), ruminal lipid synthesis (Patton et al., 1970), fiber digestion (Salsbury et al., 1971; de Vuyst et al., 1975), and shifts in VFA patterns (Lundquist et al., 1985). One such methionine analog, HMTBa, is used as a source of methionine for dairy cows (Chen et al., 2011). It has been shown that HMTBa is highly available in the rumen (Zanton et al., 2014; Koenig et al., 2002; Jones et al., 1988), but the mechanism of its action in the rumen is not completely understood. It has been reported that HMTBa may provide specific AA and peptides for cellulolytic microbes (Van Kessel and Russell, 1996) or may indirectly influence several non-cellulolytic species in the rumen.

In the current study, supplementing HMTBa to dairy cows exposed to moderate and high MFD dietary risks had a positive effect on the ruminal bacterial populations in their entirety as revealed by $\alpha$ diversity (species richness and diversity) and $\beta$ diversity (principal coordinate analysis). First, the overall bacterial species richness and diversity, which were reduced with MFD dietary risks, were mitigated with HMTBa supplementation. Reductions in richness and diversity metrics of microbial communities in the rumen have been associated with higher production efficiency in dairy cattle (Shabat et al., 2016) as well as dietary changes, although dietary changes have a significant effect on overall bacterial community and structure (Tajima et al., 2001; Henderson et al., 2015). For example, a switch to a high-grain diet (AlZahal et al., 2017) and a high-fat diet (Huws et al., 2015) have been shown to reduce overall richness and diversity. In agreement with these reports, a change in dietary composition in the current study resulted in a reduction in species richness and diversity. Although changes in bacterial species and their distribution can be influenced by several factors, the extent of variability in species richness and diversity between samples is mostly associated with significant alterations in the microbiota (Fecteau et al., 2016; Pitta et al., 2018; Stewart et al., 2019). Indeed, we found the variability between samples for species richness and diversity was greater in $\mathrm{d} 14,24$, and 28 compared with d 0 in the control group, indicating a gradual shift in bacterial communities with increasing dietary MFD risks. Interestingly, in the HMTBa group, the distribution of samples for species richness and Shannon diversity was consistent from d 0 through $d$ 28 , irrespective of the level of MFD dietary risk, indicating that HMTBa prevented aberrations in microbial community structure that are otherwise altered due to MFD dietary risks.

Second, the findings of this study support the idea that HMTBa prevents the shift to altered $\mathrm{BH}$ pathways in response to MFD dietary risks. In the study performed by M. Baldin, G. I. Zanton (USDA, Madison, WI), and K. J. Harvatine (unpublished data), the authors reported (1) the significant increase of trans-10 isomer, (2) no change in the cis-9,trans-11 CLA and trans-11 isomers, and (3) an increase in the concentrations of odd- and branched-chain fatty acids including C15:0, C17:0 isomers in the milk FA profiles of cows on the moderate- and high-risk diets without HMTBa supplementation. As described above, Dialister, Sharpea, Megasphaera, and Lachnospira were positively correlated with the trans-10 isomer, a biomarker for the altered $\mathrm{BH}$ pathway. These genera were increased in the control group but were reduced $(P<0.05)$ in the HMTBa-supplemented group on moderate- and highrisk diets compared with d 0 samples, indicating that 
HMTBa supplementation influences specific bacterial populations, particularly bacteria that are associated with the altered $\mathrm{BH}$ pathway.

Third, in agreement with the findings by Baldin et al. (2019) and M. Baldin, G. I. Zanton (USDA, Madison, WI), and K. J. Harvatine (unpublished data) that no differences were noted in cis-9,trans-11 CLA isomers between control and HMTBa groups, no bacterial populations revealed any significant positive or negative associations with this isomer. For example, the genus Butyrivibrio, known for its role in $\mathrm{BH}$ (Lourenço et al., 2010), stayed consistent between treatment groups and did not have positive or negative correlations with FA isomers. Whereas our previous report (Pitta et al., 2018) revealed positive correlations between Butyrivibrio and both cis-9,trans-11 CLA and trans10,cis-12 CLA in an induction recovery model, such patterns were not observed in this study. The relative abundance of Butyrivibrio in Pitta et al. (2018) was about $5 \%$ on the induction diet and was reduced by half to about $2.5 \%$ on the recovery diet. In this study, the relative abundance of Butyrivibrio was about $4 \%$ on $\mathrm{d} 0$ and remained at $5 \%$ on all MFD-dietary risk phases in both the control and HMTBa treatment groups, indicating that HMTBa did not influence $\mathrm{Bu}$ tyrivibrio. Higher concentrations of UFA may elevate Butyrivibrio concentrations as observed in both control and HMTBa groups to increase conversion of UFA to CLA and ultimately to vaccenic acid, as a significant percentage of the reductase required to convert CLA to vaccenic acid comes from Butyrivibrio (Hughes et al., 1982). However, it is not clear as to why HMTBa did not alter the relative abundance of Butyrivibrio in this study. Further studies are needed to understand the role of different species of Butyrivibrio in BH. Finally, we found no associations between bacterial populations and total or individuals OBCFA.

Other Firmicutes members including Veillonellaceae, Acidoaminococcus, and Shuttleworthia were positively correlated with trans-10 isomer and trans-10,cis-12 CLA isomer but were not significantly influenced by HMTBa. Actinobacteria, predominated by genus Coriobacteriaceae, showed positive correlations with trans-10,cis-12 CLA and trans-10 isomer, similar to our previous findings (Pitta et al., 2018). However, the relative abundance of Coriobacteriaceae was not statistically different between the 2 treatment groups. These findings suggest that HMTBa does not alter the entire community but may alter only the few bacteria involved in the altered pathway or those that produce the trans-10 isomer. These findings on the role of HMTBa on specific bacteria and the production of the trans-10 isomer require further research.
Notably, genera F16 (Actinobacteria), SR1 (candidate division), and $L 7 A \_E 11$ (Firmicutes) remained higher in the HMTBa group relative to the control group; however, their role in feed digestion has not been well described in the literature. The $F 16$ is positively associated with gross feed efficiency (Jewell et al., 2015), and SR1 members are known to be actively involved in sulfur metabolism (Davis et al., 2009). Although these genera decreased significantly in the control group on both moderate- and high-risk diets compared with d 0 samples, the magnitude of reduction was lower in the HMTBa group. The role of these genera in the rumen and particularly in MFD requires further investigation.

In conclusion, exposing cows gradually to increasing MFD dietary risks provided the platform to test the ability of HMTBa to alleviate signs of MFD. Supplementation with HMTBa prevented the shift to the altered $\mathrm{BH}$ pathway by modulating the ruminal bacteria in dairy cows. The MFD dietary risk factors altered the ruminal bacterial populations and increased bacteria that were positively associated with trans-10 isomer of FA, which is known to induce MFD. Supplementing HMTBa not only reduced the relative abundance of bacteria that were involved in trans-10 isomer production, but also prevented the overall shift in bacterial community profiles to those typical of cows with MFD. Further studies are needed to understand and decipher the interactions between HMTBa and rumen microbes.

\section{ACKNOWLEDGMENTS}

We are grateful to the Biomedical Research Core Facilities, University of Pennsylvania (Philadelphia), for sequencing services. The project was partially supported by Penn State University (State College) including the USDA National Institute of Food and Agriculture Federal Appropriations under project number PEN04539 and accession number 1000803, and the animal experiment was supported by Novus International (St. Charles, MO).

\section{REFERENCES}

AlZahal, O., F. Li, L. L. Guan, N. D. Walker, and B. W. McBride. 2017. Factors influencing ruminal bacterial community diversity and composition and microbial fibrolytic enzyme abundance in lactating dairy cows with a focus on the role of active dry yeast. J. Dairy Sci. 100:4377-4393. https://doi.org/10.3168/jds.2016-11473.

Anderson, M. J. 2001. A new method for non-parametric multivariate analysis of variance. Austral Ecol. 26:32-46. https://doi.org/ 10.1111/j.1442-9993.2001.01070.pp.x.

Arambel, M., E. Bartley, J. Camac, S. Dennis, T. Nagaraja, and A. Dayton. 1987. Rumen degradability and intestinal availability of a protected methionine product and its effects on rumen fermentation, and passage rate of nutrients. Nutr. Rep. Int. 
35:661-672. http://agris.fao.org/agris-search/search.do?recordID $=\mathrm{US} 874614788$.

Baldin, M., J. De Souza, E. Ticiani, E. Sandri, R. Dresch, F. Batistel, and D. Oliveira. 2017. Milk fat response to calcium salts of palm or soybean in a normal or milk fat depression scenario in dairy ewes. Livest. Sci. 206:109-112. https://doi.org/10.1016/j.livsci.2017.10 .018 .

Baldin, M., H. A. Tucker, and K. J. Harvatine. 2019. Milk fat response and milk fat and urine biomarkers of microbial nitrogen flow during supplementation with 2-hydroxy-4-(methylthio) butanoate. J. Dairy Sci. 102:6157-6166. https://doi.org/10.3168/jds.2018-15031.

Baldin, M., G. Zanton, and K. Harvatine. 2018. Effect of 2-hydroxy4-(methylthio) butanoate (HMTBa) on risk of biohydrogenationinduced milk fat depression. J. Dairy Sci. 101:376-385. https://doi .org/10.3168/jds.2017-13446.

Bauman, D. E., L. Baumgard, B. Corl, and D. J. Griinari. 1999 Biosynthesis of conjugated linoleic acid in ruminants. Proc. Am. Soc. Anim. Sci. 77:1-14. https://pdfs.semanticscholar.org/12ba/ 089f4339d9c581215066ff7574801b2c496b.pdf.

Bauman, D. E., and J. M. Griinari. 2003. Nutritional regulation of milk fat synthesis. Annu. Rev. Nutr. 23:203-227. https://doi.org/ 10.1146/annurev.nutr.23.011702.073408

Bittinger, K. 2015. qiimer: Work with QIIME output files in R. R package, Version 0.9 4. https://CRAN.R-project.org/package= qiimer.

Caporaso, J. G., K. Bittinger, F. D. Bushman, T. Z. DeSantis, G. L. Andersen, and R. Knight. 2010a. PyNAST: A flexible tool for aligning sequences to a template alignment. Bioinformatics 26:266-267. https://doi.org/10.1093/bioinformatics/btp636.

Caporaso, J. G., J. Kuczynski, J. Stombaugh, K. Bittinger, F. D. Bushman, E. K. Costello, N. Fierer, A. G. Pena, J. K. Goodrich, J. I. Gordon, G. A. Huttley, S. T. Kelley, D. Knights, J. E. Koenig, R. E. Ley, C. A. Lozupone, D. McDonald, B. D. Muegge, M. Pirrung, J. Reeder, J. R. Sevinsky, P. J. Turnbaugh, W. A. Walters, J. Widmann, T. Yatsunenko, J. Zaneveld, and R. Knight. 2010b. QIIME allows analysis of high-throughput community sequencing data. Nat. Methods 7:335-336. https://doi.org/10.1038/nmeth.f 303.

Chen, Z. H., G. Broderick, N. Luchini, B. Sloan, and E. Devillard. 2011. Effect of feeding different sources of rumen-protected methionine on milk production and N-utilization in lactating dairy cows. J. Dairy Sci. 94:1978-1988. https://doi.org/10.3168/jds.2010 -3578 .

Davis, J. P., N. H. Youssef, and M. S. Elshahed. 2009. Assessment of the diversity, abundance, and ecological distribution of members of candidate division SR1 reveals a high level of phylogenetic diversity but limited morphotypic diversity. Appl. Environ. Microbiol. 75:4139-4148. https://doi.org/10.1128/AEM.00137-09.

de Vuyst, A., M. Vanbelle, J. Joassart, and A. Baguette. 1975. The effect of methionine hydroxyanalog supplementation of the diet on the concentration of ciliate protozoa in the rumen of sheep. Z. Tierphysiol. Tierernahr. Futtermittelkd. 35:316-321. https:// doi.org/10.1111/j.1439-0396.1975.tb01106.x https://onlinelibrary .wiley.com/doi/pdf/10.1111/j.1439-0396.1975.tb01106.x.

Dewanckele, L., B. Vlaeminck, E. Hernandez-Sanabria, A. RuizGonzález, S. Debruyne, J. Jeyanathan, and V. Fievez. 2018. Rumen biohydrogenation and microbial community changes upon early life supplementation of 22:6n-3 enriched microalgae to goats. Front. Microbiol. 9:573. https://doi.org/10.3389/fmicb.2018 .00573 .

Edgar, R. C. 2010. Search and clustering orders of magnitude faster than BLAST. Bioinformatics 26:2460-2461. https://doi.org/10 .1093 /bioinformatics/btq461.

Fecteau, M. E., D. W. Pitta, B. Vecchiarelli, N. Indugu, S. Kumar, S. C. Gallagher, T. L. Fyock, and R. W. Sweeney. 2016. Dysbiosis of the fecal microbiota in cattle infected with Mycobacterium avium ssp. paratuberculosis. PLoS One 11:e0160353. https://doi.org/10 .1371/journal.pone.0160353.

Fuentes, M. C., S. Calsamiglia, P. Cardozo, and B. Vlaeminck. 2009. Effect of $\mathrm{pH}$ and level of concentrate in the diet on the production of biohydrogenation intermediates in a dual-flow continuous culture. J. Dairy Sci. 92:4456-4466. https://doi.org/10.3168/jds $2008-1722$.

Harvatine, K. J. 2016. Managing milk fat depression. Pages 64-77 in Proc. Florida Ruminant Nutr. Symp. http://dairy.ifas.ufl.edu/ rns/2016/7.\%20Harvatine.pdf.

Harvatine, K. J., Y. Boisclair, and D. Bauman. 2009. Recent advances in the regulation of milk fat synthesis. Animal 3:40-54. https://doi .org/10.1017/S1751731108003133.

Henderson, G., F. Cox, S. Ganesh, A. Jonker, W. Young, Global Rumen Census Collaborators, and P. H. Janssen. 2015. Rumen microbial community composition varies with diet and host, but a core microbiome is found across a wide geographical range. Sci. Rep. 5:14567. https://doi.org/10.1038/srep14567.

Hughes, P. E., W. J. Hunter, and S. Tove. 1982. Biohydrogenation of unsaturated fatty acids. Purification and properties of cis-9, trans11-octadecadienoate reductase. J. Biol. Chem. 257:3643-3649. http://www.jbc.org/content/257/7/3643.short.

Huws, S. A., E. J. Kim, S. J. Cameron, S. E. Girdwood, L. Davies, J. Tweed, H. Vallin, and N. D. Scollan. 2015. Characterization of the rumen lipidome and microbiome of steers fed a diet supplemented with flax and echium oil. Microb. Biotechnol. 8:331-341. https:// doi.org/10.1111/1751-7915.12164.

Huws, S. A., E. J. Kim, M. R. Lee, M. B. Scott, J. K. Tweed, E. Pinloche, R. J. Wallace, and N. D. Scollan. 2011. As yet uncultured bacteria phylogenetically classified as Prevotella, Lachnospiraceae incertae sedis and unclassified Bacteroidales, Clostridiales and Ruminococcaceae may play a predominant role in ruminal biohydrogenation. Environ. Microbiol. 13:1500-1512. https://doi.org/10 $.1111 / \mathrm{j} .1462-2920.2011 .02452 . x$.

Jenkins, T. C., R. Wallace, P. Moate, and E. Mosley. 2008. Board-invited review: Recent advances in biohydrogenation of unsaturated fatty acids within the rumen microbial ecosystem. J. Anim. Sci. 86:397-412. https://doi.org/10.2527/jas.2007-0588.

Jewell, K. A., C. A. McCormick, C. L. Odt, P. J. Weimer, and G. Suen. 2015. Ruminal bacterial community composition in dairy cows is dynamic over the course of two lactations and correlates with feed efficiency. Appl. Environ. Microbiol. 81:4697-4710. https://doi.org/10.1128/AEM.00720-15.

Jones, B. A., O. E. Mohamed, R. W. Prange, and L. D. Satter. 1988. Degradation of methionine hydroxy analog in the rumen of lactating cows. J. Dairy Sci. 71:525-529. https://doi.org/10.3168/jds .S0022-0302(88)79584-3.

Kamke, J., S. Kittelmann, P. Soni, Y. Li, M. Tavendale, S. Ganesh, P. H. Janssen, W. Shi, J. Froula, E. M. Rubin, and G. T. Attwood. 2016. Rumen metagenome and metatranscriptome analyses of low methane yield sheep reveals a Sharpea-enriched microbiome characterised by lactic acid formation and utilisation. Microbiome 4:56. https://doi.org/10.1186/s40168-016-0201-2.

Karkalas, J. 1985. An improved enzymic method for the determination of native and modified starch. J. Sci. Food Agric. 36:1019-1027. https://doi.org/10.1002/jsfa.2740361018.

Kim, Y. J., R. H. Liu, J. L. Rychlik, and J. B. Russell. 2002. The enrichment of a ruminal bacterium (Megasphaera elsdenii YJ-4) that produces the trans-10, cis-12 isomer of conjugated linoleic acid. J. Appl. Microbiol. 92:976-982. https://doi.org/10.1046/j.1365-2672 .2002.01610.x

Koenig, K. M., L. M. Rode, C. D. Knight, and M. Vázquez-Añón. 2002. Rumen degradation and availability of various amounts of liquid methionine hydroxy analog in lactating dairy cows. J. Dairy Sci. 85:930-938. https://doi.org/10.3168/jds.S0022-0302(02)74151 -9 .

Latham, M. J., J. E. Storry, and M. E. Sharpe. 1972. Effect of lowroughage diets on the microflora and lipid metabolism in the rumen. Appl. Microbiol. 24:871-877. https://www.ncbi.nlm.nih.gov/ pmc/articles/PMC380689/pdf/applmicro00052-0045.pdf.

Lee, C., J. Oh, A. Hristov, K. Harvatine, M. Vazquez-Anon, and G. Zanton. 2015. Effect of 2-hydroxy-4-methylthio-butanoic acid on ruminal fermentation, bacterial distribution, digestibility, and performance of lactating dairy cows. J. Dairy Sci. 98:1234-1247. https://doi.org/10.3168/jds.2014-8904. 
Lobley, G. E., T. Wester, A. G. Calder, D. Parker, J. Dibner, and M. Vázquez-Añón. 2006. Absorption of 2-hydroxy-4-methylthiobutyrate and conversion to methionine in lambs. J. Dairy Sci. 89:10721080. https://doi.org/10.3168/jds.S0022-0302(06)72175-0.

Lourenço, M., E. Ramos-Morales, and R. Wallace. 2010. The role of microbes in rumen lipolysis and biohydrogenation and their manipulation. Animal 4:1008-1023. https://doi.org/10.1017/ S175173111000042X.

Lundquist, R. G., M. Stern, D. Otterby, and J. Linn. 1985. Influence of methionine hydroxy analog and DL-methionine on rumen protozoa and volatile fatty acids. J. Dairy Sci. 68:3055-3058. https://doi .org/10.3168/jds.S0022-0302(85)81201-7.

Maia, M. R., L. C. Chaudhary, C. S. Bestwick, A. J. Richardson, N. McKain, T. R. Larson, I. A. Graham, and R. J. Wallace. 2010. Toxicity of unsaturated fatty acids to the biohydrogenating ruminal bacterium, Butyrivibrio fibrisolvens. BMC Microbiol. 10:52. https://doi.org/10.1186/1471-2180-10-52.

Maia, M. R. G., L. C. Chaudhary, L. Figueres, and R. J. Wallace. 2007. Metabolism of polyunsaturated fatty acids and their toxicity to the microflora of the rumen. Antonie van Leeuwenhoek 91:303-314.

Mandal, S., W. Van Treuren, R. A. White, M. Eggesbø, R. Knight, and S. D. Peddada. 2015. Analysis of composition of microbiomes: A novel method for studying microbial composition. Microb. Ecol. Health Dis. 26:27663. https://doi.org/10.3402/mehd.v26.27663.

Martin, C., C. Mirande, D. Morgavi, E. Forano, E. Devillard, and P. Mosoni. 2013. Methionine analogues HMB and HMBi increase the abundance of cellulolytic bacterial representatives in the rumen of cattle with no direct effects on fibre degradation. Anim. Feed Sci. Technol. 182:16-24. https://doi.org/10.1016/j.anifeedsci.2013 .03 .008 .

Martińez, I., D. J. Perdicaro, A. W. Brown, S. Hammons, T. J. Carden, T. P. Carr, K. M. Eskridge, and J. Walter. 2013. Dietinduced alterations of host cholesterol metabolism are likely to affect the gut microbiota composition in hamsters. Appl. Environ. Microbiol. 79:516-524.

McDonald, D., M. N. Price, J. Goodrich, E. P. Nawrocki, T. Z. DeSantis, A. Probst, G. L. Andersen, R. Knight, and P. Hugenholtz. 2012. An improved Greengenes taxonomy with explicit ranks for ecological and evolutionary analyses of bacteria and archaea. ISME J. 6:610-618. https://doi.org/10.1038/ismej.2011.139.

Mohammed, R., D. M. Stevenson, K. A. Beauchemin, R. E. Muck, and P. J. Weimer. 2012. Changes in ruminal bacterial community composition following feeding of alfalfa ensiled with a lactic acid bacterial inoculant. J. Dairy Sci. 95:328-339. https://doi.org/10 $.3168 /$ jds.2011-4492.

Palmonari, A., D. M. Stevenson, D. R. Mertens, C. W. Cruywagen, and P. J. Weimer. 2010. $\mathrm{pH}$ dynamics and bacterial community composition in the rumen of lactating dairy cows. J. Dairy Sci. 93:279-287. https://doi.org/10.3168/jds.2009-2207.

Patton, R. A., R. McCarthy, and L. C. Griel Jr.. 1970. Observations on rumen fluid, blood serum, and milk lipids of cows fed methionine hydroxy analog. J. Dairy Sci. 53:776-780. https://doi.org/10 .3168/jds.S0022-0302(70)86289-0.

Pitta, D. W., N. Indugu, B. Vecchiarelli, D. Rico, and K. Harvatine. 2018. Alterations in ruminal bacterial populations at induction and recovery from diet-induced milk fat depression in dairy cows. J. Dairy Sci. 101:295-309. https://doi.org/10.3168/jds.2016-12514.

Price, M. N., P. S. Dehal, and A. P. Arkin. 2010. FastTree 2-approximately maximum-likelihood trees for large alignments. PLoS One 5:e9490. https://doi.org/10.1371/journal.pone.0009490.

R Core Team. 2019. R: A language and environment for statistical computing. https://www.r-project.org/.

Rico, D. E., and K. J. Harvatine. 2013. Induction of and recovery from milk fat depression occurs progressively in dairy cows switched between diets that differ in fiber and oil concentration. J. Dairy Sci. 96:6621-6630. https://doi.org/10.3168/jds.2013-6820.

Rico, D. E., S. Preston, J. Risser, and K. Harvatine. 2015. Rapid changes in key ruminal microbial populations during the induction of and recovery from diet-induced milk fat depression in dairy cows. Br. J. Nutr. 114:358-367. https://doi.org/10.1017/ S0007114515001865.

Rico, D. E., Y. Ying, A. Clarke, and K. Harvatine. 2014. The effect of rumen digesta inoculation on the time course of recovery from classical diet-induced milk fat depression in dairy cows. J. Dairy Sci. 97:3752-3760. https://doi.org/10.3168/jds.2013-7342.

Salsbury, R. L., D. Marvil, C. Woodmansee, and G. Haenlein. 1971. Utilization of methionine and methionine hydroxy analog by rumen microorganisms in vitro. J. Dairy Sci. 54:390-396. https://doi .org/10.3168/jds.S0022-0302(71)85850-2.

Shabat, S. K. B., G. Sasson, A. Doron-Faigenboim, T. Durman, S. Yaacoby, M. E. B. Miller, B. A. White, N. Shterzer, and I. Mizrahi. 2016. Specific microbiome-dependent mechanisms underlie the energy harvest efficiency of ruminants. ISME J. 10:2958-2972.

Song, S. J., C. Lauber, E. K. Costello, C. A. Lozupone, G. Humphrey, D. Berg-Lyons, J. G. Caporaso, D. Knights, J. C. Clemente, S. Nakielny, J. I. Gordon, N. Fierer, and R. Knight. 2013. Cohabiting family members share microbiota with one another and with their dogs. eLife 2:e00458. https://doi.org/10.7554/eLife.00458.

Stewart, H. L., L. L. Southwood, N. Indugu, B. Vecchiarelli, J. B. Engiles, and D. Pitta. 2019. Differences in the equine faecal microbiota between horses presenting to a tertiary referral hospital for colic compared with an elective surgical procedure. Equine Vet. J. 51:336-342. https://doi.org/10.1111/evj.13010.

Sukhija, P. S., and D. Palmquist. 1988. Rapid method for determination of total fatty acid content and composition of feedstuffs and feces. J. Agric. Food Chem. 36:1202-1206.

Tajima, K., R. I. Aminov, T. Nagamine, H. Matsui, M. Nakamura, and Y. Benno. 2001. Diet-dependent shifts in the bacterial population of the rumen revealed with real-time PCR. Appl. Environ. Microbiol. 67:2766-2774. https://doi.org/10.1128/AEM.67.6.2766 $-2774.2001$.

Van Kessel, J. S., and J. Russell. 1996. The effect of amino nitrogen on the energetics of ruminal bacteria and its impact on energy spilling. J. Dairy Sci. 79:1237-1243. https://doi.org/10.3168/jds.S0022 -0302(96)76476-7.

Van Soest, P. J., J. Robertson, and B. Lewis. 1991. Methods for dietary fiber, neutral detergent fiber, and nonstarch polysaccharides in relation to animal nutrition. J. Dairy Sci. 74:3583-3597.

Wallace, R. J., N. McKain, K. J. Shingfield, and E. Devillard. 2007. Isomers of conjugated linoleic acids are synthesized via different mechanisms in ruminal digesta and bacteria. J. Lipid Res. 48:2247-2254. https://doi.org/10.1194/jlr.M700271-JLR200.

Weimer, P. J., D. M. Stevenson, and D. R. Mertens. 2010. Shifts in bacterial community composition in the rumen of lactating dairy cows under milk fat-depressing conditions. J. Dairy Sci. 93:265278. https://doi.org/10.3168/jds.2009-2206.

Yu, Z., and M. Morrison. 2004. Comparisons of different hypervariable regions of rrs genes for use in fingerprinting of microbial communities by PCR-denaturing gradient gel electrophoresis. Appl. Environ. Microbiol. 70:4800-4806.

Zanton, G. I., G. R. Bowman, M. Vázquez-Añón, and L. M. Rode. 2014. Meta-analysis of lactation performance in dairy cows receiving supplemental dietary methionine sources or postruminal infusion of methionine. J. Dairy Sci. 97:7085-7101. https://doi.org/10 $.3168 /$ jds.2014-8220.

Zened, A., S. Combes, L. Cauquil, C. Rousseau, C. Klopp, A. Troegeler-Meynadier, and F. Enjalbert. 2011. The ruminal level of trans-10 fatty acids of dairy cows is linked to the composition of bacterial community. In 4th Congress of European Microbiologists, Geneva, Switzerland.

\section{ORCIDS}

D. W. Pitta @ https://orcid.org/0000-0002-3102-9119 M. Hennessy @ https://orcid.org/0000-0002-1297-7623

M. Baldin (๑ https://orcid.org/0000-0003-4875-1404

K. J. Harvatine ㄴ) https://orcid.org/0000-0001-6422-2647 